\title{
Mirror, Mirror, on the Wall, Which Is the Most Effective Biologic of All?
}

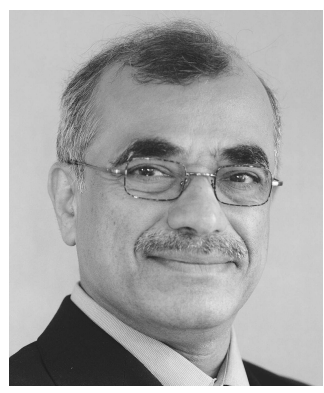

We all consider the "comparative effectiveness" of available treatment options in daily clinical practice, knowingly or unknowingly. In fact, that and "comparative safety" are 2 of our most important and constant considerations when planning the best possible therapy for any disorder in rheumatology. In daily practice, clinicians mostly depend on their personal experience and knowledge, because head-to-head randomized controlled trials (RCT) are not available for most of the medications we use, and for most of the rheumatologic conditions we treat. Introduction of biologic therapy to treat immune-mediated inflammatory conditions has made this task even more challenging, because these agents have proven to be generally very effective but potentially toxic, and they are uniformly expensive. Which biologic should we choose to treat a patient with active rheumatoid arthritis, psoriatic arthritis, or ankylosing spondylitis (AS)? Several times, the economic realities of health insurance coverage tie our hands and choose the biologic therapy for us, and in the absence of scientific data on comparative effectiveness or safety, it is difficult to argue one way or the other. Treatment guidelines developed by national or international societies play a big role in helping us choose the right treatment, and comparative effectiveness is considered formally with statistical analysis or informally with expert opinion when developing such guidelines. Several statistical methods are available for assessing comparative effectiveness. Treatment guidelines by the American College of Rheumatology use network metaanalysis (NMA), whereas the UK National Institute for Health and Care Excellence, which decides the national formulary, prefers Matching Adjusted Indirect Comparison (MAIC) for assessing the comparative effectiveness and safety of therapies for the same indication.

NMA and MAIC are methods for indirect comparison of the efficacy of 2 drugs when no direct comparison is available. NMA considers efficacy of drug A and drug B versus a common comparator - usually placebo; and then uses pooled OR for drug A and B versus placebo, or effect sizes for drug $\mathrm{A}$ and $\mathrm{B}$, for such indirect comparison. The advantage of NMA methodology is that it maintains the benefits of randomization, and combines only relative treatment effects. The disadvantage of NMA is that it assumes that the RCT are homogeneous. If a treatment effect modifier, for example baseline $\mathrm{C}$-reactive protein (CRP) in AS trials, is very different in trials of drug A and trials of drug $\mathrm{B}$, then the indirect comparison between $\mathrm{A}$ and $\mathrm{B}$ can become very biased. MAIC avoids this problem, because it applies propensity score matching to adjust individual patient-level data to a given patient population. The disadvantage of MAIC is that, in the process of propensity score matching, it reduces the effective sample size.

This issue of The Journal has an important article by Wang, et al on comparative effectiveness of tumor necrosis factor inhibitors (TNFi) in the treatment of AS using NMA ${ }^{1}$. This was a large undertaking, because including a biosimilar, we now have $6 \mathrm{TNFi}$ available to treat AS in North America. To assess the efficacy of each TNFi versus placebo, this group carried out a systematic literature review of all available clinical trials on each TNFi against placebo. Then they used Bayesian NMA to compare the effectiveness of 5 "originator" and 1 biosimilar TNFi. They used 3 continuous variables - Bath Ankylosing Spondylitis Disease Activity Index (BASDAI), Bath Ankylosing Spondylitis Functional Index, and CRP as outcome measures to assess the efficacy of these agents. The choice of continuous variables as outcome measures was deliberate because previous attempts assessing comparative effectiveness of TNFi in AS used the Assessment of Spondyloarthritis international Society guidelines (ASAS20), a variable that is dichotomous and hence less sensitive to change. Wang and colleagues found that infliximab (IFX) was the most effective TNFi in the treatment of AS, though this was true only for a single endpoint - BASDAI at Week 12; when an open-label trial on IFX was omitted, this advantage was lost.

Studies like this are going to be progressively important for making clinical decisions in daily practice as different classes of agents become available to treat AS. With the approval of interleukin 17 inhibitors, the question of comparative effectiveness and safety has become very pertinent.

See TNF- $\alpha$ inhibitors in ankylosing spondylitis, page 481

Personal non-commercial use only. The Journal of Rheumatology Copyright (C) 2018 . All rights reserved. 
There have been several previous studies on this subject. Chen, et al carried out a systematic literature review and NMA to assess the comparative effectiveness of not only $\mathrm{TNFi}$, but also tocilizumab and secukinumab in the treatment of AS with ASAS20 response as the outcome of interest ${ }^{2}$. Like the Wang, et al's study published in this issue, they too found IFX to be most efficacious, followed by secukinumab. Lee and Song assessed the comparative efficacy and safety of secukinumab versus adalimumab (ADA) in the treatment of AS by Bayesian $\mathrm{NMA}^{3}$. Using the surface under the cumulative ranking curve, they found that ADA was a better treatment for achieving an ASAS20 response than secukinumab, and there was no difference in safety in these 2 agents.

Manufacturers of biologics not surprisingly prefer this statistical exercise for indirect comparison rather than potentially risky and very expensive head-to-head RCT. They have sponsored several studies on comparative effectiveness and safety between their own drug with a competitor biologic. All such studies that included IFX showed superiority of IFX in comparative efficacy, but also showed some favorable results for the manufacturer's own biologic over the comparators. A study from the manufacturers of secukinumab investigated the comparative effectiveness of secukinumab with all TNFi using $\mathrm{NMA}^{4}$. In that study, IFX had the best efficacy, but secukinumab was statistically superior to etanercept, ADA, and certolizumab for improving BASDAI. A study sponsored by manufacturers of ADA compared ADA with 4 other TNFi and secukinumab ${ }^{5}$. They found that IFX had the lowest numbers needed to treat to achieve an additional ASAS20 and ASAS40 response, but ADA had the lowest cost per ASAS20 and ASAS40 responder among biologic agents for the treatment of active AS. Two recent studies have compared ADA with secukinumab in the treatment of AS by MAIC ${ }^{6,7}$. These studies used the same baseline data and showed similar results of comparable efficacy between ADA and secukinumab up to the placebo-controlled period (12 weeks and 16 weeks, respectively). However, the study sponsored by the manufacturers of ADA showed that the incremental costs per respondent, relative to placebo, were lower for ADA compared to secukinumab across all efficacy measures ${ }^{6}$. The study sponsored by the manufacturers of secukinumab reported higher ASAS20 response rates at weeks 16, 24, and 52 for secukinumab compared to ADA, though this result was not placebo-adjusted ${ }^{7}$. Similarly, comparative effectiveness of secukinumab and golimumab in the treatment of AS was assessed by MAIC in a study sponsored by the manufacturer of secukinumab ${ }^{8}$. No differences in ASAS responses were found between the 2 drugs in placebo-adjusted analyses, but in non-placebo-adjusted analyses, secukinumab showed higher ASAS20 and 40 responses compared to golimumab at Week 24.

Comparative effectiveness and safety studies are essential to make informed decisions regarding appropriate treatment in daily practice, and studies such as that of Wang, et al are therefore important. It is hoped that payers would allow the use of the most efficacious and safest treatment rather than forcing treatment decisions based solely on the cost.

\section{ATUL DEODHAR, MD \\ Professor of Medicine, \\ Oregon Health \& Science University, Portland, Oregon, USA.}

Address correspondence to Dr. A. Deodhar, Professor of Medicine, Division of Arthritis \& Rheumatic Diseases (OP09), Oregon Health \& Science University, 3181 SW Sam Jackson Park Road, Portland, Oregon 97239, USA.E-mail: deodhara@ohsu.edu

\section{REFERENCES}

1. Wang R, Dasgupta A, Ward MM. Comparative efficacy of tumor necrosis factor- $\alpha$ inhibitors in ankylosing spondylitis: a systematic review and Bayesian network metaanalysis. J Rheumatol 2018;45:481-90.

2. Chen C, Zhang X, Xiao L, Zhang X, Ma X. Comparative effectiveness of biologic therapy regimens for ankylosing spondylitis: a systematic review and a network meta-analysis. Medicine 2016;95:e3060.

3. Lee HO, Song GG. Comparative efficacy and safety of secukinumab and adalimumab in patients with active ankylosing spondylitis: a Bayesian network meta-analysis of randomized controlled trials. J Rheum Dis 2017;24:211-9.

4. Baeten D, Mease P, Strand V, McInnes I, Thom H, Kanters S, et al. SAT0390 Secukinumab for the treatment of ankylosing spondylitis: comparative effectiveness results versus currently licensed biologics from a network meta-analysis [poster presentation]. Ann Rheum Dis 2016;75:809-10.

5. Betts K, Griffith J, Song Y, Mittal M, Joshi A, Wu EQ, et al. Network meta-analysis and cost per responder of tumor necrosis factor- $\alpha$ and interleukin inhibitors in the treatment of active ankylosing spondylitis. Rheumatol Ther 2016;3:323-36.

6. Betts KA, Mittal M, Song J, Skup M, Joshi A. OP0115 Relative efficacy of adalimumab versus secukinumab in active ankylosing spondylitis: a matching-adjusted indirect comparison [oral presentation]. Ann Rheum Dis 2016;75:98-9.

7. Maksymowych WP, Strand V, Nash P, Thom H, Karabis A, Gandhi $\mathrm{K}$, et al. Comparative effectiveness of secukinumab and adalimumab in ankylosing spondylitis as assessed by matching-adjusted indirect comparison: an analysis based on all pivotal phase 3 clinical trial data [abstract]. Arthritis Rheumatol 2016;68 Suppl 10:1739.

8. Maksymowych WP, Choy E, Yazici Y, Walsh JA, Thom H, Kalyvas $\mathrm{C}$, et al. Comparative effectiveness of secukinumab and golimumab in ankylosing spondylitis: assessed by matching-adjusted indirect comparison using pivotal phase 3 clinical trial data [abstract]. Arthritis Rheumatol 2017;69 Suppl 10:1546.

J Rheumatol 2018;45:449-50; doi:10.3899/jrheum.171279

Personal non-commercial use only. The Journal of Rheumatology Copyright $\odot$ (2018. All rights reserved 